\title{
Analysis of Dosage Form of Traditional Chinese Medicine
}

\author{
Huiyuan Huang ${ }^{1, a}$ and Mingsan Miao ${ }^{1, b, *}$ \\ ${ }^{1}$ Pharmacological disciplines, Henan University of Chinese Medicine, Zheng Zhou, Henan, China \\ ayaolihuanghuiyuan@126.com, bmiaomingsan@163.com \\ *corresponding author
}

Keywords: Traditional Chinese Medicine, Formulation, Related Problems, Solutions

\begin{abstract}
This paper traces the history of traditional Chinese medicine treatment formulations, introduces the characteristics of traditional Chinese medicine external treatment dosage forms and modern commonly used external treatment dosage forms, and puts forward the external treatment of traditional Chinese medicine formulations on the problems and future development prospects.
\end{abstract}

\section{Introduction}

The history of dosage form of traditional Chinese medicine is very long.External application agent, lotion, Paste, Liniment, powder, Medicinal strip preparation, Moxapreparation, Film, cataplasm, Adhesive plasters, Targeting preparation, Topical sustained release film, Ion introduction agents and other forms are emerging from ancient times to the present. Chinese medicine external dosage form system is also developing and improving, but there are still some serious problems that we urgently need to solve. This paper analyses the history of traditional Chinese medicine dosing, the characteristics of ancient and modern dosage forms and their applications to point out the problems that we should pay attention to and the prospects for development in the future.

\section{The Historical Traceability of Traditional Chinese Medicine}

External treatment of traditional Chinese medicine has a long history. The earliest records of traditional Chinese medicine on the ancient books should be Shan Hai Jing, the book recorded that yellow irrigation bath could treat scabies. Zhou Li-Tian Gong mentioned external medicine could cure sore. So as early as thousands of years ago there were topical formulations to treat skin diseases. During the Spring and Autumn period and the Warring States period, lotion appeared. Shan Hai Jing refers to the paint, Pei, bath, nourishing and so on, bath and paint are said that the bathing agent and liniment. There are paste, fumigant, bath and smoke agent in Prescriptions of Fifty-two Diseases, the earliest extant book in China [1], and put forward the application time, issues related to dietary taboos impact on drug safety [2]. Prescriptions of Fifty-two Diseases also mentioned a large number of drug-related content. For example, infant disease in epileptic seizures can be recorded in the bath. Huang Di Nei Jing refers to the external dosage forms, such as powder, ointment, iron and so on. Canon of Medicine marks the further development of the external treatment system. Shennong Bencao Jing, the earliest Chinese materia medica monograph, discussed the relationship between drugs and dosage forms and explains its importance in the clinical application. The book also mentioned some specific forms of drugs. Bezoar should be made into pills. Angelica should be boiled. To sum up, the external governance theory at this time is only in the embryonic stage.

The descendants are based on the experience of predecessors, and the theory of external governance is developing rapidly. Ge Hong, a famous medical writer in the Eastern Jin Dynasty, wrote various kinds of dosage forms in Zhou Hou Fang. There are pills, cream, wine, bolt, wash, rub, ointment, sachet and pillow more than 10 kinds of formulations, pills are the most varieties, including honey pill, bitter pill, medicine pill and so on. Powder is second only to pills and pastes. The paste is the second largest dosage form, fried cream, plaster, ointment are mostly modern use of various plaster prototype. Shanghan Zabing Lun is the earliest 
recorded suppository, on the basis of which also invented the nasal suppository, ear bolt, vaginal suppository, etc., belongs to the ancient books that contain the largest suppository. The Northern and Southern Dynasties, Liu Juan-zi's Gui-Yi-fang recorded of topical paste 79 and details of its preparation. Sushen Liang Fang discussed the choice of dosage forms in clinic. Sui and Tang Dynasties were the most flourishing period of prescription development. Sniffing agent, melting agent, nasal suction agent and other dosage forms appear [3]. In the Tang Dynasty, Valuable Prescriptions for Emergency written by drug king Sun Simiao recorded paste, bath, milk, mu, mud, smoked, juice and so on [4]. In addition, the Sui and Tang dynasties also invented many Zijin paste, Taiyi cream and other umbilical treatment plaster, continues today. In Song Dynasty, external system was more abundant, Ju Fang which is China's first proprietary Chinese medicine specification formed in this period. The forms of book are versatile, pills, loose, decoction-based, followed by ointment, snow agent, lozenges, fragrances and other agents [5]. By the Ming Dynasty, the development of medicine reached the highest level in history. Compendium of Materia Medica is a representative work of this period. Dosage forms are rich in this book, each with its own characteristics, such as strips, powder, suppository, plaster, plaster, ointment, liniment, gargle and so on. Ointment, suppository and other dosage forms are now still widely used, which had a profound impact on the development of modern medicine. A large number of books published in the Qing Dynasty rule, external treatment of traditional Chinese medicine theory system was more perfect and formed a certain scale. The preparation method and treatment theory of plaster were discussed in detail in Li Yue Pianwen. Jiju Guangsheng Ji and Li Yue Pianwen marked the external treatment of traditional Chinese medicine thought gradually mature.

\section{Traditional Chinese Medicine Formulation and Characteristics}

There are many traditional Chinese medicine topical formulations. More common as follows:

(1) the powder is a powder preparation in which the medicinal materials are pulverized and mixed uniformly after sieving. Powder can be divided into oral, external use of two kinds. The Canon of Internal Medicine, Prescriptions of Fifty-two Diseases, Treatise on Febrile Diseases and Synopsis of Golden Chamber are recorded. The utility model has the advantages of convenient carrying, simple preparation method, no dosage requirement, large coverage area, quick curative effect, and the efficacy of convergence and hemostasis.

(2) The liniment can be divided into solution type liniment and suspension type liniment. Solution type liniment means that the drug is dissolved in a certain proportion of the solvent. Ethanol, glycerin aqueous solution, dimethyl sulfoxide, and fatty oil are often the components of the solution type liniment. The utility model has the advantages of wide application range. The solution type liniment has the efficacy of protection, convergence and sterilization. Suspension liquid Liniment (water powder, lotion), an external preparation made from $30 \% \sim 50 \%$ insoluble powder (such as talcum powder) in water. As early as in the Spring and Autumn Warring States period, there appeared a lotion. Glycerin is often added to make it better. Its characteristics are as follows: stable, cheap, simple process, moisturizing, isolation to reduce external irritation to skin, skin itching, detoxification effect. Such as anti itching lotion with a wind dampness itching, convergence, detoxification [6]. Chinese medicine zhitongxiao anorectal disease Postoperative Infection Prevention Lotion [7].

(3) The drug added to the preparation and then made the appropriate matrix topical semi-solid or solid, it is called mastic. Lubrication, protection, local treatment or the role of systemic treatment are the main feature. The drug directly into the systemic circulation through the skin to avoid the liver first pass effect and drug destruction in the gut, so it has very few side effects. The paste is divided into ointment, plaster and so on.

(4) Moxa-preparation, the treatment is China old treatment methods, using the "hot" physical therapy. The Golden Mirror of Medicine contained roast moxibustion in Qing Dynasty. Leaves and other drugs are rolled together into a cigarette - like to use to smoke the diseased parts, is called moxibustion agent. it can soothe the muscular region and dredge collaterals, replenishing vitality, regulate qi and blood, prevent disease.

(5) Compression formula. Drug extract, vinegar, calcined sand mixed and then dry to made topical 
preparations. The principle of iron treatment of the disease is the use of chemical reactions generated by the heat stimulation and herbs vapor into the iron paste the site of pain to achieve blood circulation, divergent treatment of the purpose of cold [8]. The preparation has the advantages of simple and convenient preparation, cheap material, easy preservation, no other side effects, etc.

(6) Pastille, a solid or semisolid dosage form of different shapes made from a drug powder or binder. The lozenges have two methods of kneading and molding. Zhouhou Beiji Fang recorded in the preparation of lozenges were more drugs and fine with vinegar, such as zijin ding. Modern use of lozenges is more extensive. For example, lozenges on the cervical lymph nodes, osteomyelitis, sore ulcers have therapeutic effect. Lozenges are easy to carry and store, easy to use (grinding juice outside the affected area) [9].

(7) Suppository (suppository, suppository) refers to the cavity to solid preparation of drugs. The medicine is solid at room temperature and dissolves into the cavity and slowly releases the drug, thereby producing therapeutic action. A long time ago at home and abroad had recorded suppository. In $1500 \mathrm{BC}$, the ancient Egyptian Yiboshi Zhicaoen mentioned suppositories. Shiji Canggong Biography, Treatise on Febrile Diseases and Zhouhou Beiji Fang have a lot of records about the contents of suppositories. Suppositories in the cavity can produce a series of local therapeutic effects, such as pain, antibacterial, itching, antiinflammatory, etc., for example, snake bolt. Suppositories can also be directly absorbed by the rectal mucosa into the blood to produce analgesia, sedation systemic therapy, for example, morphine supository. The suppository is absorbed directly in the cavity, which is not disturbed by the gastrointestinal tract $\mathrm{pH}$ or enzyme, reduces the drug to the gastrointestinal tract stimulation, avoids the first pass effect, reduces the toxic and side effects, and brings great convenience to the patient who can not take medicine.

(8) Medicated roll, it also called medicine twist in ancient times. Different materials twist into a thin strip or gauze wrapped in drugs made of the preparation known as the article. The use of the physical effect of thin strip to remove the role of detoxification. Preparation of the method is simple, good efficacy, as early as in clinical applications. Hongshengdan and Baijiangdan made twisted bars treatment of green snake venom and irrigation twist governance hemorrhoids. That is recorded in The Golden Mirror of Medicine and Yangyi Daquan. Various types of agents, paper twist, fiber twist, hard strip, soft cloth, gauze bar. As a black tiger Dan hard twist dispel rotten pus swelling, Salvia miltiorrhiza gauze cloth antibacterial and anti-inflammatory.

\section{Modern topical dosage forms}

With the continuous development of modern science and technology, people's understanding of the dosage forms has been further improved, and many new forms of dosage forms have been developed and perfected on the basis of traditional dosage forms. Modern commonly used external dosage forms are as follows:

(1) Aerosol is a combination of drugs and propellants in a pressure vessel, and the aerosol is ejected by the pressure of the propellant. It has the characteristics of quick positioning, high stability, accurate dosage, convenient use and little side effect, but its effect is easily affected by the properties of drugs, the size of fog particles and the state of inhalation. It is mainly used for inhalation of the lungs and into the mucous membrane or skin of the cavity and often used in combination with other drugs. Salbutamol aerosol combined with montelukast sodium chewable tablets can treat children with asthma [10]. Fluticasone Propionate Aerosol may be the treatment of allergic rhinitis [11]. Salbutamol sulfate aerosol can treat acute elderly asthma [12].

(2) The production process of film agent is simple, there are many ways of administration, the dosage is small, the content of drug is accurate, the efficacy is long, the film forming material is different, the speed of drug release is different. It is used for the treatment of vaginitis, skin and mucous membrane, burns, inflammation and other diseases. Compound Bifonazole Pigment can treat tinea manus and pedis [13]. Topical chitosan spray can be used to treat pediatric burns [14].

(3) Gel is new formulations in recent years, it is thick liquid or semi solid preparation. There are latex, mucilage, suspension gel. Because the gel is biocompatible, gel can control the drug release rate. Its characteristics are as follows: good stability, easy coating, not easy to pollute clothes, good curative effect, 
long acting time and the local absorption is easy to absorb. Preparation of gel with high quality requirements, such as material and drug reaction does not occur, the gel is uniform, delicate and so on. Gel has six routes of administration for transdermal, oral, ocular, nasal, vaginal and rectal administration. Gel is mainly used for skin fungal or viral infections, acne, induced labor, contraception, rhinitis, oral ulcers, and dental inflammation.

(4) Babu is a new topical patch. At present, the research on Babu agent in our country is gradually deepened, and the quality control index and dosage form evaluation system has been improved. Babu has the characteristics of large amount of drugs, fast penetration, high bioavailability, convenient use, safe and effective. The structure mainly consists of three parts: support layer, paste layer and backing layer. It is used for pain caused by diseases such as arthritis, bone hyperplasia, abdominal cavity and cancer. For example, babu, a blood pain inhibitor, has anti-inflammatory and analgesic effects [15].

(5) There are two kinds of rubber plasters: medicated and non medicated. Rubber paste should be smooth, no paste phenomenon. Plaster has strong adhesive force, can be directly attached to the skin, and no pollution of clothes, but because the plaster layer is thin, relatively short effect. The principle of pharmacodynamics is to produce therapeutic effect by transdermal absorption, which is used to treat arthralgia and sprain caused by rheumatic diseases. In addition, external dosage forms include targeted preparations, transdermal controlled release preparations, topical sustained-release membranes, iontophoresis agents, and so on. Such as Fu Le Ning sustained-release film can treat vaginitis, the medicine membrane is widely distributed and cooperative interaction, fast absorption, good effect, safety, no irritation. Zhang Yuqian used the compound honeysuckle iontophoresis agents for the treatment of acute conjunctivitis, stye and other diseases.

\section{The current topical dosage form should pay attention to the problem}

Modern preparation technology in China has been developing vigorously, and a lot of new preparations and new formulations have appeared. However, there are many problems with external dosage forms, and they are facing great challenges. In order to make our pharmaceutical industry develop more effectively, we should pay more attention to the following problems when preparing external dosage forms.

(1) Form rationality. The declaration of mutual modification of dosage forms is increasing in new form of declaration, such as tincture or ointment to spray, lotion or paste to ointment. The variety of dosage forms is to make more choices in clinical application, but we should consider the rationality of the dosage form, consider whether the suitability of clinical indications and the needs of patients.

(2) Process variation. Is there a qualitative change in the manufacturing process after the dosage form changes? In the declaration, it is expressly stated that the formulation of the dosage form is changed only when the route of administration is constant. At present, many forms of change after the declaration is no qualitative change. We should consider formulation changes in matrix and excipients on drug absorption and utilization, indications, whether the safety and effectiveness of the impact.

(3) Dosage. External preparation dose is uncertain in use, such as a patch, powder, paste. Some drugs contain toxic components, and if the dose is not used accurately, it can affect people's health. We should consider the dose of the drug and how to give more accurate dose of administration.

(4) Safety. Some special delivery sites have particular requirements for the condition of certain drugs in the dosage form. For example, the cavity or mucosal suppository supplements, the preparation of certain drugs in the powder will cause the drug in the cavity or mucous membrane in the local residual, affecting its physiological function. Therefore, in the development of the cavity or mucosal administration of the preparation, it is not appropriate to raw materials such as borneol raw powder added directly to the preparation. Special attention should be paid to external use preparations containing large toxic Chinese medicine. When used in the wound, the skin is easy to absorb the toxic components, resulting in adverse reactions. Therefore, when developing a toxic component containing a local preparation, we should consider its indications, the characteristics of the site, the use of penetrating agent is reasonable and other factors [16]. 


\section{Prospect}

As for the rationality of the dosage forms, we should make a detailed analysis of the specific problems according to the clinical needs, the characteristics of the illness and the personal constitution. In order to select a dosage form that is more suitable for patient rehabilitation, we should sometimes consider pharmacokinetics in vivo and pharmacological actions in the selection of dosage forms. For the preparation process of the dosage form, the government strengthens the process supervision, the researchers optimize the process and put forward the theoretical basis of the process optimization, and examine the process parameters so that the active ingredients of the drug are extracted more. For the dose problems, we should increase the study of the relevant dose pharmacological experiments, explore the best safe dose, optimize the production process, research and development more safe, effective and appropriate new formulations. For security issues, the relevant government departments should increase supervision. In conclusion, we developed the future direction of topical formulations and new formulation of joint efforts is to explore more ways of administration, seek external treatment for internal diseases, improve the bioavailability of drugs, the drug toward safety, cheap and efficient way.

\section{Acknowledgment}

Fund project: the national international cooperation base (State University letter 2016-65), the Central Plains scholars (162101510003), Henan Province, production and research projects (142201310013, 172107000012).

\section{References}

[1] Chen Renze. Literature and Theoretical Research on Zhang Zhongjing's External Treatment [D]. Beijing University of Chinese Medicine, 2010.

[2] Niu Hongjuan, Zhang Bing, Lin Zhijian, Zhou Jun, Zhang Xiaomeng, Sun Xiaoxia. Analysis of safe medication based on the history of external treatment of Chinese medicine [J]. Applied Medicine and Clinical, 2015, (12): 1501-1504.

[3] Xu Xia. A Historical Study on the Formulation of Traditional Chinese Medicine [D]. Chinese Academy of Sciences, 2010.

[4] Sun Zhanxue. Chinese medicine outside the rule of law and the source [A]. Chinese Medicine Association of Dermatology. 2013 Chinese Medicine Association of Dermatology Branch of the tenth academic exchange conference and Hunan Province, the combination of Chinese and Western medicine sexually transmitted diseases, the eighth academic exchange conference papers [C]. Chinese Medicine Association of Dermatology Branch, 2013: 3.

[5] Wu Chengyan. Analysis of the Masterpieces of the Taiping Huimin Heji Ju Fang in the Song Dynasty [J]. Shi Zhenguo Medicine, 2012, (12): 3088-3089.

[6] Chen Mingling, Ai Hua editor. Contemporary Chinese medicine dermatology clinical series of books Ai Ru Di [M]. Beijing. China Medical Science and Technology Press, 2014. 107-110

[7] Feng Liuquan, Ye Feng, Song Weiping, Sun Xiaochao, Zhang Ying. Chinese medicine lotion on the prevention of postoperative anorectal infection [J]. Chinese Journal of Nosocomiology, 2013, (18): 4347.

[8] WU Wei, Miao Mingsan. Characteristics and Application of Commonly Used Traditional Chinese Medicine Formulations [J]. Chinese Journal of Traditional Chinese Medicine, 2011, (01): 108-110.

[9] Fu Chaomei, Liu Wen editor, Chinese medicine pharmacy [M]. Beijing. China Medical Science and Technology Press, 2014. 323-324

[10] Zhang Yanhong, Zhang Chao. Effect of salbutamol aerosol combined with montelukast sodium chewable tablet in the treatment of children with asthma [J]. Clinical Medicine Research and Practice, 2017, (10): 45-46.

[11] Tan Junwu, Liao Yong, Peng Hong, Li Liangbo, Sun Shuju, Yu Fang Fang. Clinical study of fluticasone 
propionate aerosol on children with allergic rhinitis [J]. Chinese Journal of Clinical Pharmacology, 2017, (06): 561-564.

[12] Ye Chunjuan, Zhang Guoyu. Comparative analysis of the effect of salbutamol aerosol inhalation and theophylline on the treatment of acute elderly asthma [J]. Chinese Journal of Biochemical Pharmaceutics, 2014, (01): 116-117+ 120 .

[13] Li Miao, Li Shide, Shao Mingxian, Xi, Qiu Youlu, Li Huaizhang. Preparation and clinical application of compound bifonazole coating agent [J]. Chinese Journal of Hospital Pharmacy, 2009, (09): 757-758.

[14] Chen Zhiyuan, Cao Weipeng, Shi Fei, Liu Qinghuan.Comparison of topical chitosan spray agent and MEBO in pediatric scald treatment [J]. Jilin Medical, 2015, (18): 4111-4112.

[15] Hou Jun, Dou Jianwei, Li Jing, Liu Aiqin, You Fengming, Zheng Dezhi, Li Dandan, Zhou Longfu, Hu Yonghe. Study on the pharmacodynamics of anti-inflammatory and analgesic effects of Zhizhi prescription [J]. Journal of Southeast China National Defense Medical Science, 2017, (01): 8-12.

[16] Jin Fang. Attention should be paid to the research and quality evaluation of external preparations of traditional Chinese medicine in [J]. China Journal of TCM, 2010, (21): 2934-2936. 